\title{
Modelling and control for bounded synchronization in multi-terminal VSC-HVDC transmission networks
}

\author{
Arnau Dòria-Cerezo, Josep M. Olm, Member, IEEE, Mario di Bernardo, Fellow, IEEE, and Emmanuel Nuño
}

\begin{abstract}
The extension and size of the power grid is expected to increase in the near future. Managing such a system presents challenging control problems that, so far, have been approached with classical control techniques. However, large scale systems of interconnected nodes fall within the framework of the emerging field of complex networks. This paper models multiterminal VSC-HVDC systems as a complex dynamical network, and derives conditions ensuring bounded synchronization of its trajectories for a family of controllers. The obtained results are validated via numerical simulations.
\end{abstract}

Keywords-HVDC transmission, complex networks, bounded synchronization, droop control.

\section{INTRODUCTION}

Achieving efficient energy transport and distribution has emerged as an important problem for the future. New technologies, most of them related to renewable energies, have changed the structure and methods of generation, management and power consumption.

The potential of High Voltage Direct Current (HVDC) transmission has been lately boosted by the development of Voltage-Source Converters (VSC) based on HVDC systems. Indeed, among the advantages of VSC technology with respect to classical current-fed line-commutated converters are: an independent control of the reactive and active power consumed or generated by the converter, smaller filter size due to a faster dynamic response, and no need of transformers to assist the commutation process [1], [2].

In turn, technical, economical and environmental reasons make HVDC lines a feasible alternative to High Voltage Alternating Current (HVAC) lines, if not the only option, for long distance bulkpower dispatch, power transmission in asynchronous interconnection situations, or long submarine cable crossings. This guarantees grid access to renewable sources such as large-scale wind farms, hydro-electric facilities, or mine-mouth power plants in remote areas. In particular, the

\footnotetext{
A. Dòria-Cerezo is with the Department of Electrical Engineering and the Institute of Industrial and Control Engineering, Universitat Politècnica de Catalunya, 08028 Barcelona, Spain.

J.M. Olm is with the Department of Mathematics and the Institute of Industrial and Control Engineering, Universitat Politècnica de Catalunya, 08028 Barcelona, Spain.

M. di Bernardo is with the Department of Electrical Engineering and Information Technologies, University of Naples Federico II, 80125 Naples, Italy, and with the Department of Engineering Mathematics, University of Bristol, BS8 1UB Bristol, U.K.

E. Nuño is with the Department of Computer Science, CUCEI, University of Guadalajara, 44430 Guadalajara, Mexico.

E-mails: arnau.doria@upc.edu, josep.olmeupc.edu, mario.dibernardo@unina.it, emmanuel.nuno@cucei.udg.mx.
}

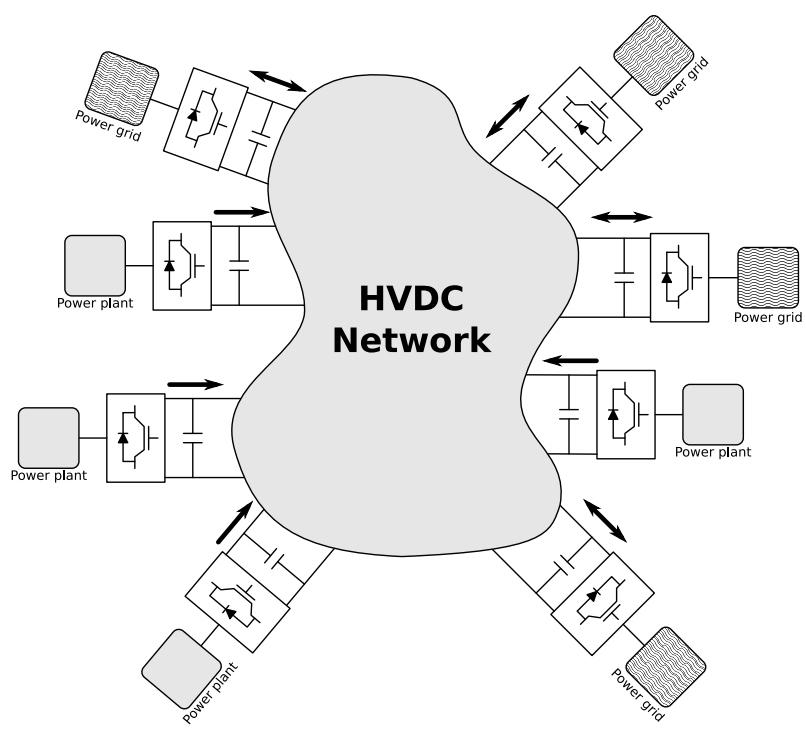

Fig. 1: Multi-terminal HVDC transmission scheme.

use of HVDC links in offshore wind farms improves voltage and frequency responses independently of the wind turbine type, and alleviates the effect of grid faults [3], [4].

Different power plants are connected to the power grid side stations through a meshed DC grid. In such schemes, known as Multi-terminal HVDC (M-HVDC) networks [2], terminals are power generation plants and grid connection stations, as illustrated in Fig. 1. Nevertheless, although M-HVDC grids are undoubtedly a much more efficient alternative to pointto-point connections, they also present many challenges from the control perspective. For example, a primary goal for an M-HVDC power grid is to ensure power balance and to keep voltages in a certain range under faulty conditions. This requirement is met in recent designs by using droop control [5]. For instance, droop control is used in [6] as a decentralized voltage regulator, while in [7] it is exploited to ensure both stability and an acceptable performance in an M-VSC HVDC transmission system for offshore wind power plants. The analysis carried out in [5] is based on linearization, thus providing local results. Instead, [7], [8] studies global stability through a 
static output feedback approach based on an $L_{2}$ optimization criterion solved through Linear Matrix Inequalities (LMIs). This technique shows three main drawbacks: firstly, it neglects constant or quasi-constant signals, for which the $L_{2}$ space is not an appropriate framework; secondly, the computational burden associated to LMI solving prevents its application to MHVDC systems with a high number of terminals; and, thirdly, it does not provide error bounds for the state variables.

To overcome these problems, a different approach is required. Specifically, a strategy needs to be devised to deal with a large ensemble of interconnected nodes coupled through a given topological structure and guarantee that they exhibit some desired macroscopic properties. This is precisely the type of problem studied in network control. Indeed, complex networks have been attracting much research interest since the end of last century, and many cooperative problems arising in natural, engineering and social sciences have been tackled within such a framework [9], [10], [11]. A typical example is synchronization [11], [12], [13], [14], [15]: a specific type of collective behaviour emerging when the state trajectories of the nodes asymptotically tend towards each other. Static synchronization, i.e. asymptotic convergence to a common equilibrium, is known as consensus [11], [16], [17]. While either consensus or synchronization are realistic goals in networks with identical nodes, such a behavior is in general impossible in networks with nonidentical nodes. In this case, an asymptotically bounded mismatch can be achieved between the states and a certain "reference" trajectory, a regime often termed as bounded synchronization [18], [13].

Large-scale M-HVDC transmission systems can be regarded as complex dynamical networks [9], [10], so can the power grid itself [19]. The terminals, each one with its own dynamics, can be modelled as the nodes of the network, and the connecting lines as the coupling edges. However, most papers in the literature dealing with power grids using a complex network approach [19] focus on frequency synchronization in $\mathrm{AC}$ grids [20], [21], [22], [23], [24], [25]. A recent work dealing with voltage synchronization of coupled power electronics inverters [26] was further extended to more general classes of nonlinear oscillators [27], but networks of identical nodes were considered in both cases. It is also worth mentioning that a cooperative droop control strategy for DC microgrids, which could fall within complex networks protocols, was proposed in [28]; nevertheless, the stability analysis is again based on LMIs. Finally, a decentralized PI passivity-based controller that renders global asymptotic stability for VSCHVDC transmission networks has been recently presented in [29]; however, current and voltage saturation effects are not considered.

This paper models an M-VSC HVDC transmission system as a complex network with nonidentical nodes, where a current source (for power generating terminals) or sink (for power consuming terminals) in each node acts as the control action. Then, the problem of ensuring power balance and maintaining bounded voltages during faults is reformulated as that of achieving bounded synchronization. In so doing, the occurrence of bounded synchronization in resistive and ResistiveCapacitive-Inductive (RLC) transmission lines is ensured for a family of controllers which encompass droop control laws while taking into account saturation constraints; explicit error bounds are also computed. Numerical results on a realistic scenario with power availability and power demand varying in time validate the theoretical predictions.

The remainder of the paper is organized as follows. A basic background in complex networks is given in Section II. Section III includes a mathematical model of M-VSC HVDC transmission systems and is cast into a complex network fashion. Sections IV and V contain, respectively, the bounded synchronization analysis for the resistive and RLC case. $\mathrm{Nu}-$ merical results are shown in Section VI, and conclusions are drawn in Section VII.

\section{COMPLEX NETWORKS}

We summarize next some preliminary results on complex networks that are mostly taken from [9], [18].

We consider undirected, complex dynamical networks of $n$ non-identical, one-dimensional nodes with linear diffusive coupling, modeled by:

$$
\dot{x}_{k}=f_{k}\left(x_{k}, t\right)+\sum_{l=1}^{n} a_{k l}\left(x_{l}-x_{k}\right), \quad k=1, \ldots, n
$$

where $x_{k} \in \mathbb{R}$ is the state of the $k$-th node, $f_{k}: \mathbb{R} \times \mathbb{R} \rightarrow \mathbb{R}$ is a piecewise continuous map, and $a_{k l}$ is the coefficient of the weighted adjacency matrix, that is, $a_{k l}>0$ if nodes $k$ and $l$ are coupled.

The weighted Laplacian matrix $\Lambda=\left(\Lambda_{k l}\right) \in \mathbb{M}_{n}(\mathbb{R})$, defined via the weighted adjacency matrix as

$$
\Lambda_{k l}= \begin{cases}\sum_{l=1}^{n} a_{k l} & k=l \\ -a_{k l} & k \neq l\end{cases}
$$

allows to write (1) in matrix form as:

$$
\dot{x}=f(x, t)-\Lambda x,
$$

with $x=\left(x_{1}, \ldots, x_{n}\right)^{\top}$ and $f=\left(f_{1}, \ldots, f_{n}\right)^{\top}$.

The Laplacian matrix satisfies the following properties:

P1 It has zero row-sum: $\sum_{l} \Lambda_{k l}=0, k=1, \ldots, n$.

P2 $\mathbf{1}_{n}=(1, \ldots, 1)^{\top} \in \mathbb{R}^{n}$ is a right eigenvector of $\Lambda$ with eigenvalue 0 , i.e. $\Lambda \mathbf{1}_{n}=0$.

Let now $r(t)=\left(r_{1}(t), \ldots, r_{n}(t)\right)^{\top}$, with $r_{i}: \mathbb{R}_{\geq 0} \longrightarrow \mathbb{R}$, $i=1, \ldots, N$, denote a certain reference trajectory.

Definition 1: Network (1) is said to achieve bounded synchronization with respect to some reference trajectory $r$ if there exists $\epsilon>0$ such that

$$
\lim _{t \rightarrow+\infty}\|x(t)-r(t)\| \leq \epsilon
$$

\section{MUlTi-TERMinAl VSC-HVDC TRANSMISSION SYSTEMS}

\section{A. Control architecture}

An M-VSC HVDC transmission system is composed of a number of power plants connected to a main AC power grid through a meshed DC grid, with VSC converters transforming the DC electrical power into an AC waveform, or vice-versa. 


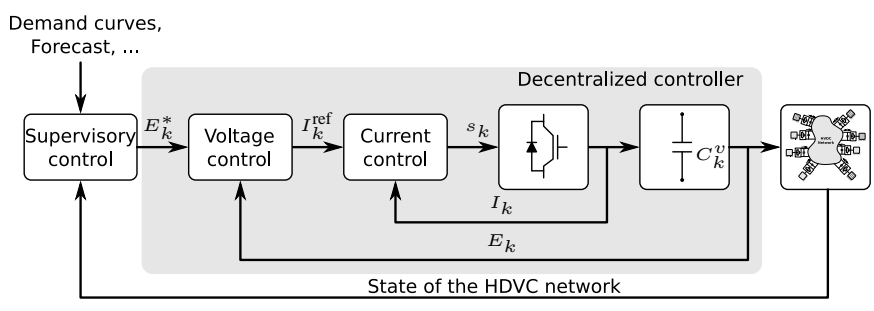

Fig. 2: Control scheme.

The control strategy for these networks is based on a hierarchical, three control level architecture [6], see Fig. 2. This scheme consists of: a supervisor algorithm that sets the required voltages, $E_{k}^{*}$, to all VSC converters; a voltage control scheme that regulates the voltages of each VSC capacitor; and a current controller providing the switching policy to inject/extract the required current, $I_{k}$, to/from the capacitor, $C_{k}^{v}$. Such a current is obtained from the voltage controller, which is the focus of this paper.

\section{B. Mathematical model}

A VSC converter can be modeled as a current source in parallel with a capacitor [6], [7], see Fig. 3. The current in terminal $k, I_{k}$, takes positive values when power is being injected into the DC grid, and negative values otherwise. $I_{k}$ is typically positive in power generating terminals and negative in power consuming terminals, though changes of role, and therefore, of the current sign, are allowed when required for power balance.

The current supplied by each VSC, $I_{k}\left(E_{k}, t\right)$, is used to regulate the corresponding DC voltage, $E_{k}(t)$, taking into account the power supplied (or consumed) by the power plant (or AC grid). During operation, the characteristic curve $\phi_{k}^{E}:=$ $\left(E_{k}(t), I_{k}\left(E_{k}, t\right)\right)$ must remain within an admissibility region in the $\left(E_{k}, I_{k}\right)$ state plane, denoted as $\mathcal{I}_{k}^{E}$, corresponding to the area limited by the maximum and minimum allowed voltages, $E_{k}^{\max }$ and $E_{k}^{\min }$, respectively, the rated power of the power converter, $P_{k \mathrm{I}}^{\max }$ (injecting) and $P_{k \mathrm{C}}^{\min }$ (consuming), and the boundary values for the current, $I_{k}^{\max }$ and $I_{k}^{\min }$, see Fig. 4.

As mentioned above, the most common control technique for the VSC in an HVDC network is droop voltage control,

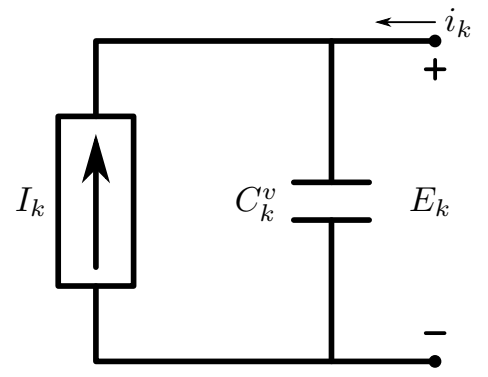

Fig. 3: Equivalent circuit of a VSC.

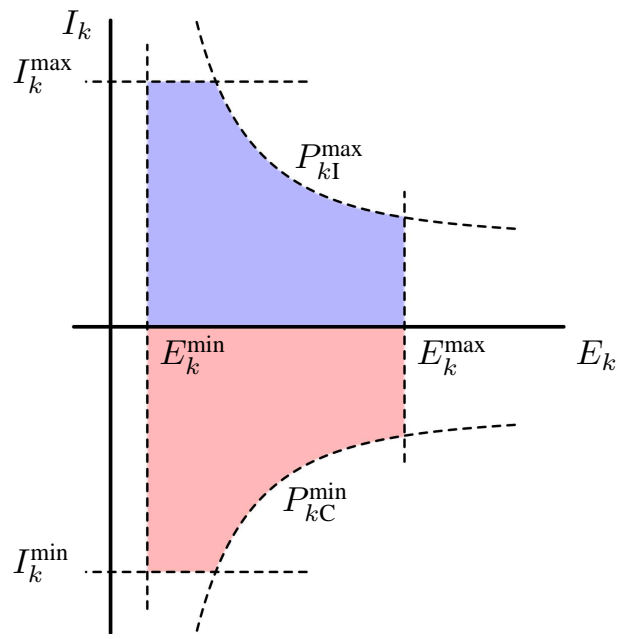

Fig. 4: Admissibility region for $\phi_{k}^{E}:=\left(E_{k}, I_{k}\left(E_{k}\right)\right)$. Blue area: power injection; red area: power consumption.

which basically consists of a static curve $I_{k}\left(E_{k}\right)$ that maintains maximum power (normal operation in power plants) for a certain range of $E_{k}$, and switches to proportional gain when required (normal operation in AC grid side converters), see the example in [7]. However, here we assume that a generic, nonlinear, static control current, $I_{k}=I_{k}\left(E_{k}\right)$, is employed.

A lumped parameter transmission line model is used to describe the link between two nodes, $k$ and $l$, see Fig. 5 .

The dynamics of both VSC and DC transmission lines are obtained from Kirchhoff's Currents Law (KCL) and Kirchhoff's Voltages Law (KVL). Applying KCL to Fig. 3, the dynamics of each VSC is given by

$$
C_{k} \frac{\mathrm{d} E_{k}}{\mathrm{~d} t}=I_{k}\left(E_{k}\right)+i_{k},
$$

where $E_{k}$ is the voltage at the capacitor $C_{k}^{v}, C_{k}$ is the equivalent capacity at node $k$, i.e.

$$
C_{k}=C_{k}^{v}+\frac{1}{2} \sum_{l=1}^{n} C_{k l},
$$

and $I_{k}\left(E_{k}\right)$ is the current injected (or consumed) by the power converter. As mentioned above, the $I_{k}$ value is used to regulate

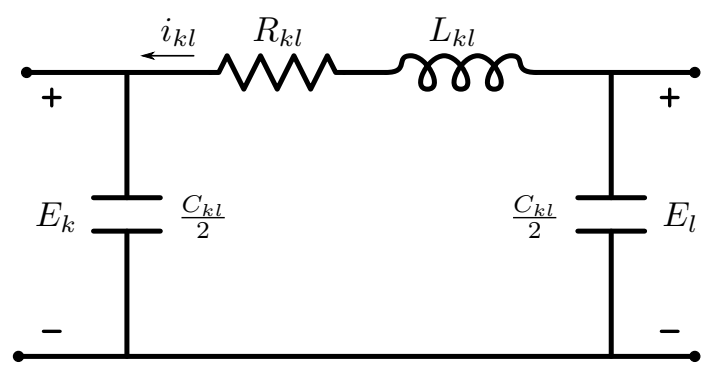

Fig. 5: Equivalent circuit of a transmission line. 
the voltage $E_{k}$ within an appropriate range, and $I_{k}\left(E_{k}\right)$ is assumed to be a nonlinear static relationship. The incoming current into the $k$-th VSC node, $i_{k}$, can be computed as

$$
i_{k}=\sum_{l=1}^{n} a_{k l} i_{k l}
$$

where $i_{k l}$ is the current flowing from node $l$ to node $k$ (see Fig. 5), and

$$
a_{k l}= \begin{cases}1 & \text { if } l \text { and } k \text { are connected } \\ 0 & \text { if } l \text { and } k \text { are not connected. }\end{cases}
$$

From Fig. 5, and using KVL, the dynamics of a transmission line connecting nodes $k$ and $l$ can be derived as

$$
E_{l}=E_{k}+R_{k l} i_{k l}+L_{k l} \frac{\mathrm{d} i_{k l}}{\mathrm{~d} t},
$$

where $R_{k l}$ and $L_{k l}$ are the resistance and inductance of each line, respectively. Notice that $i_{k l}=-i_{l k}$.

\section{M-VSC HVDC transmission systems as complex dynamical networks}

A multi-terminal HVDC transmission system can then be seen as a complex dynamical network where the nodes are the VSC of each power plant (or power grid connection) and the communication protocol is given by the topology of the transmission line.

However the DC power network will probably contain star nodes, i.e. nodes not corresponding to power terminals but to points where two or more transmission lines are connected, as in the example depicted in Fig. 6a. To resolve this problem Kron's reduction [30], [31] can be used, which allows to obtain an equivalent network with all nodes corresponding to power terminals or grid connections. As pointed out in [31], Kron's reduction is applicable to pure resistive networks and also to homogeneous RLC networks, i.e. networks satisfying $R_{k l} L_{k l}^{-1}=R_{k l} C_{k l}^{-1}=c$, for all $(k, l)$ such that $a_{k l} \neq 0$. Notice that this constraint may be overcome using transmission lines with the same resistance, inductance, and capacitance per unit length. Finally, it is worth pointing out that homogeneity is required because the analysis encompasses not only steadystate but also transient behavior [31].

Hence, the M-VSC HVDC transmission system model considered here consists of a set of nodes (plants and power grid converters) connected through a point-to-point undirected protocol (power transmission lines). This yields an undirected complex dynamical network as shown in Fig. 6b [9].

Let us now derive the network dynamics model. Letting $m \in$ $\mathbb{N}$ stand for the total number of transmission lines, we define the line currents vector $i=\left(i_{k l}\right) \in \mathbb{R}^{m}$, where $k, l=1, \ldots, n$ with $k<l$ and $a_{k l} \neq 0$. It is immediate that $m \leq \frac{n(n-1)}{2}$. Then, from (5) the network node dynamics can be written in a matrix form as

$$
C \frac{\mathrm{d} E}{\mathrm{~d} t}=-A i+I(E)
$$

where $E=\left(E_{1}, \ldots, E_{N}\right)^{\top} \in \mathbb{R}^{N}$ is the voltage vector, $I(E)=\left(I_{1}\left(E_{1}\right), \ldots, I_{N}\left(E_{N}\right)\right)^{\top} \in \mathbb{R}^{N}$ is the control currents

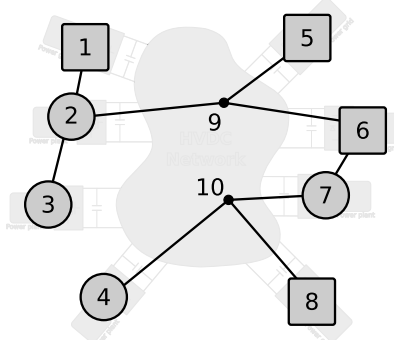

(a)

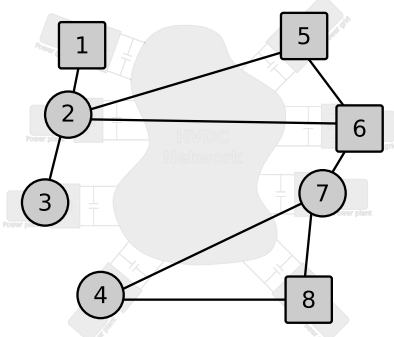

(b)
Fig. 6: M-HVDC transmission system (a) with star nodes; (b) mesh-transformed. Power plants and grid side stations are represented by circles and squares, respectively.

vector, $C=\operatorname{diag}\left(C_{i}\right) \in \mathbb{R}^{n \times n}$, and $A=\left(A_{k j}\right) \in \mathbb{R}^{n \times m}$ is the incidence matrix:

$$
A_{k j}=\left\{\begin{aligned}
1 & \text { if line } j \text { is outgoing from node } k \\
-1 & \text { if line } j \text { incoming into node } k, \\
0 & \text { otherwise. }
\end{aligned}\right.
$$

Equivalently, defining the $\mathbb{M}_{m}(R)$ inductance and resistance matrices $L=\operatorname{diag}\left(L_{k l}\right), R=\operatorname{diag}\left(R_{k l}\right)$, respectively, the transmission lines dynamics (9) can be written as:

$$
L \frac{\mathrm{d} i}{\mathrm{~d} t}=-R i+A^{\top} E .
$$

Furthermore, $R$ being a non-singular matrix one can isolate $i$ in (11) and substitute it into (10). Thus, the overall system dynamics is given by

$$
\begin{aligned}
C \frac{\mathrm{d} E}{\mathrm{~d} t} & =I(E)+A R^{-1} L \frac{\mathrm{d} i}{\mathrm{~d} t}-G E, \\
L \frac{\mathrm{d} i}{\mathrm{~d} t} & =-R i+A^{\top} E,
\end{aligned}
$$

with $G:=A R^{-1} A^{\top}$ being the conductance matrix.

Assume that the voltage reference for node $k$ is set to $E_{k}^{*}$, obviously with

$$
E_{k}^{\min }<E_{k}^{*}<E_{k}^{\max },
$$

and define the error gap as:

$$
e_{k}=E_{k}-E_{k}^{*} \text {. }
$$

We also assume $E^{*}:=\left(E_{k}^{*}\right)^{\top} \notin \operatorname{span}\left\{\mathbf{1}_{n}\right\}$ so as to avoid setting all nodes to the same voltage, where there is no power flow. Hence, (12)-(13) become:

$$
\begin{aligned}
C \frac{\mathrm{d} e}{\mathrm{~d} t} & =I(e)+A R^{-1} L \frac{\mathrm{d} i}{\mathrm{~d} t}-G E^{*}-G e, \\
L \frac{\mathrm{d} i}{\mathrm{~d} t} & =-R i+A^{\top} e+A^{\top} E^{*},
\end{aligned}
$$

with $I(e)=\left(I_{1}\left(e_{1}\right), \ldots, I_{N}\left(e_{N}\right)\right)^{\top} \in \mathbb{R}^{N}$.

Notice that the node equation (16) matches the generic model (3) of an undirected network, with

$$
\begin{aligned}
f(e, t) & =C^{-1} I(e)+C^{-1} A R^{-1} L \frac{\mathrm{d} i}{\mathrm{~d} t}-C^{-1} G E^{*}, \\
\Lambda & =G,
\end{aligned}
$$




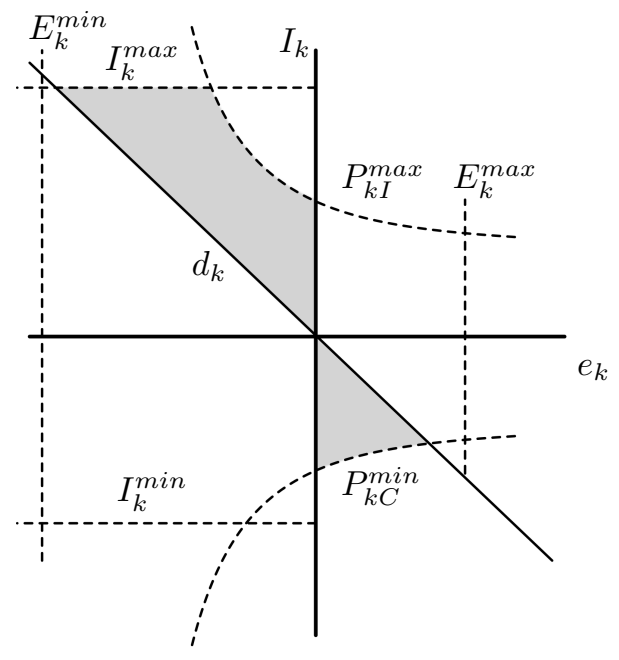

Fig. 7: Grey area: the $k$-th control admissibility region.

$G$ being a weighted Laplacian, while (17) stands for the edge dynamics. Hence, the M-HVDC grid has in fact dynamic interconnections [27], and can be modelled as an adaptive network, see [32], [33] for further details.

Finally, recall from Section III-B that the current at every node, $I_{k}\left(E_{k}\right)$, must be such that the characteristic curve $\phi_{k}^{E}$ belongs to an admissible set $\mathcal{I}_{k}^{E}$ of the plane $\left(E_{k}, I_{k}\right)$; we denote as $\phi_{k}^{e}:=\left(e_{k}(t), I_{k}(t)\right)$ and $\mathcal{I}_{k}^{e}$ the corresponding characteristic curve and admissible region, respectively, in the $\left(e_{k}, I_{k}\right)$ plane. Let us now introduce a second admissibility region for $\phi_{k}^{e}$ :

$$
\mathcal{J}_{k}^{e}:=\left\{\left(e_{k}, I_{k}\right) ;\left(\begin{array}{c}
I_{k} \geq-d_{k} e_{k} \text { if } e_{k} \leq 0 \\
I_{k} \leq-d_{k} e_{k} \text { if } e_{k}>0
\end{array}\right)\right\},
$$

with

$$
d_{k} \geq d_{k}^{*}>0, \quad \forall k
$$

Definition 2: A current control vector $I(e)$ for network (16)-(17) is said to be admissible if it is such that, for every node $k$, the characteristic curve $\phi_{k}^{e}$ belongs to $\mathcal{I}_{k}^{e} \cap \mathcal{J}_{k}^{e}, \forall t \geq 0$. In turn, $\mathcal{I}_{k}^{e} \cap \mathcal{J}_{k}^{e}$ is termed as the $k$-th control admissibility region.

The $k$-th control admissibility region $\mathcal{I}_{k}^{e} \cap \mathcal{J}_{k}^{e}$ is depicted in Fig. 7.

\section{RESISTIVE M-HVDC TRANSMisSiON SYSTEMS}

A purely resistive model is obtained by setting $C_{k l}=0$ in (6) and $L_{k l}=0$ in (9). Hence, (16)-(17) boil down to:

$$
C \frac{\mathrm{d} e}{\mathrm{~d} t}=I(e)-G e-G E^{*} .
$$

Let us define the parameter $e^{*}$ as:

$$
e^{*}:=\frac{1}{2} E^{* \top} G E^{*} .
$$

It has been assumed in Section III-C that the voltage references are not the same for every node, i.e. $E^{*} \notin \operatorname{span}\left\{\mathbf{1}_{n}\right\}$; this, together with the weighted Laplacian character of $G$, yields $e^{*} \in \mathbb{R}^{+}$. Finally, let $\Omega$ be a hyperellipsoid of the $n$-th dimensional error space centered in $e=0 \in \mathbb{R}^{n}$ and defined as:

$$
\Omega:=\left\{e \in \mathbb{R}^{n} ; e^{\top} C e \leq s^{* 2}\right\}
$$

with

$$
s^{*}:=\sqrt{e^{*} \max \left\{\frac{C_{k}}{d_{k}}, k=1, \ldots, N\right\}} .
$$

As shown below sufficient conditions can be established to ensure that the terminal voltages evolve within or towards $\Omega$.

Theorem 1: Assume that the control current vector $I(e)$ in (20) is admissible. If $e(0) \in \Omega$, then $e(t) \in \Omega, \forall t \geq 0$; otherwise, if $e(0) \in \mathbb{R}^{n} \backslash \Omega$, then $e(t)$ approaches $\bar{\Omega}$ as $t \rightarrow+\infty$.

Proof: Let us consider the following positive definite auxiliary function:

$$
V(e)=\frac{1}{2} e^{\top} C e .
$$

Its derivative over the system trajectories is:

$$
\dot{V}(e)=e^{\top} C \dot{e}=e^{\top} I(e)-e^{\top} G e-e^{\top} G E^{*} .
$$

The positive semidefiniteness of $G$ entails:

$0 \leq\left(e+E^{*}\right)^{\top} G\left(e+E^{*}\right)=e^{\top} G e+E^{* \top} G E^{*}+2 e^{\top} G E^{*} ;$ hence, taking into account (21) yields:

$$
-e^{\top} G E^{*} \leq \frac{1}{2} e^{\top} G e+\frac{1}{2} E^{* \top} G E^{*}=\frac{1}{2} e^{\top} G e+e^{*} .
$$

Moreover, it is immediate from the definition of $\mathcal{J}_{k}^{e}$ in (18) that, $\forall k \in\{1, \ldots, n\}, \phi_{k}^{e} \subset \mathcal{J}_{k}^{e} \Longrightarrow I_{k} e_{k}^{2} \leq-d_{k} e_{k}^{2}$. Therefore, defining $D:=\operatorname{diag}\left(d_{k}\right) \in \mathbb{M}_{n}(\mathbb{R})$, one has that

$$
e^{\top} I(e) \leq-e^{\top} D e,
$$

with $D$ positive definite and $e^{*}>0$. Consequently, from (24) it follows:

$$
\begin{aligned}
\dot{V}(e) & \leq-e^{\top} D e-e^{\top} G e+\frac{1}{2} e^{\top} G e+e^{*}= \\
& =-e^{\top} D e-\frac{1}{2} e^{\top} G e+e^{*} \leq-e^{\top} D e+e^{*} .
\end{aligned}
$$

Let us now define the hyperellipsoid

$$
\widehat{\Omega}:=\left\{e \in \mathbb{R}^{n} ; e^{\top} D e \leq e^{*}\right\} .
$$

Notice that $e=0 \in \widehat{\Omega}$, and also that $\dot{V} \leq 0$ in $\partial \widehat{\Omega}$ and $\dot{V}<0$ in $\mathbb{R}^{n} \backslash \widehat{\Omega}$. Moreover, under the change of coordinates

$$
\bar{e}:=\frac{1}{\sqrt{2}} C^{\frac{1}{2}} e,
$$

with $C^{\frac{1}{2}}=\operatorname{diag}\left(\sqrt{C_{k}}\right)$ being the principal square root of $C$, $\widehat{\Omega}$ becomes a hyperellipsoid in the $\bar{e}$-state space, its maximum semi-axis being $\frac{s^{*}}{\sqrt{2}}$, with $s^{*}$ defined in (23). As the surfaces of the $\bar{e}$-state space with constant $V(\bar{e})$ are now hyperspheres, the one with radius $\frac{s^{*}}{\sqrt{2}}$ is the smallest one enclosing $\widehat{\Omega}$; in the $e$-state space, such an $\mathbb{R}^{n}$ subset is precisely $\Omega$, defined 
in (22). Thus, $\Omega$ is a closed set where $V(\partial \Omega)$ is constant, $\dot{V}(\partial \Omega) \leq 0$, and $\dot{V}\left(\mathbb{R}^{n} \backslash \Omega\right)<0$. Hence, the result follows.

Remark 1: According to (22), the lengths of the semi-axes of the hyperellipsoid $\Omega, s_{k}$, are given by

$$
s_{k}:=\frac{s^{*}}{\sqrt{C_{k}}}=\sqrt{\frac{e^{*}}{C_{k}} \max \left\{\frac{C_{l}}{d_{l}}, l=1, \ldots, n\right\} .}
$$

Therefore, the size of the subset of $\mathbb{R}^{n}$ where the evolution of the system is confined in (or towards which it approaches), depends on the slopes, $d_{k}$, on the capacitor values, $C_{k}$, and on the voltage setpoint references, $E_{k}^{*}$ (see (21)): higher slopes and capacitors, and closer (but not identical) voltage references correspond to smaller hyperellipsoids, and vice-versa. Notice, finally, that restriction (19) ensures that $\Omega$ has a bounded size.

Corollary 1: Assume that the control current vector $I(e)$ in (20) is admissible. Then, network (20) achieves bounded synchronization with respect to $r(t)=0$. Specifically,

$$
\lim _{t \rightarrow+\infty}\|e(t)\| \leq \epsilon=\max \left\{s_{k}, k=1, \ldots, n\right\} .
$$

\section{RLC M-HVDC TRANSMISSION SYSTEMS}

In this case the edge dynamics are not neglected, so the overall network dynamics is given by (16)-(17).

Let $\Omega_{R L C}$ be the following hyperellipsoid of the $(n+m)$-th dimensional error space, centered in $\left(e^{\top}, i^{\top}\right)^{\top}=0 \in \mathbb{R}^{n+m}$ and defined as:

$$
\Omega_{R L C}:=\left\{\left(e^{\top}, i^{\top}\right)^{\top} \in \mathbb{R}^{n+m} ; e^{\top} C e+i^{\top} L i \leq s_{R L C}^{* 2}\right\},
$$

where

$$
s_{R L C}^{*}:=\max \left\{s^{*}, \sqrt{2} s_{L}^{*}\right\},
$$

with $s^{*}$ defined in (23) and

$$
s_{L}^{*}:=\sqrt{e^{*} \max \left\{\frac{L_{k}}{R_{k}}, k=1, \ldots, m\right\}},
$$

$e^{*}$ being that defined in (21).

The analogous result of Theorem 1 for the RLC case is as follows:

Theorem 2: Assume that the control current vector $I(e)$ in (16)-(17) is admissible. If $\left(e^{\top}(0), i^{\top}(0)\right)^{\top} \in \Omega_{R L C}$, then $\left(e^{\top}(t), i^{\top}(t)\right)^{\top} \in \Omega_{L}, \forall t \geq 0$; otherwise, if $\left(e^{\top}(0), i^{\top}(0)\right)^{\top} \in \mathbb{R}^{N} \backslash \Omega_{R L C}$, then $\left(e^{\top}(t), i^{\top}(t)\right)^{\top}$ approaches $\Omega_{R L C}$ as $t \rightarrow+\infty$.

Proof: Let us consider the following auxiliary function:

$$
V(e, i)=\frac{1}{2} e^{\top} C e+\frac{1}{2} i^{\top} L i .
$$

Using (16)-(17), the time derivative of $V(e, i)$ over the system trajectories can be written as:

$$
\dot{V}(e, i)=e^{\top} I(e)-i^{\top} R i+i^{\top} A^{\top} E^{*} .
$$

Now, letting $R^{\frac{1}{2}}$ stand for the principal square root of $R$, one has that

$$
\begin{aligned}
0 & \leq\left(R^{\frac{1}{2}} i-R^{-\frac{1}{2}} A^{\top} E^{*}\right)^{\top}\left(R^{\frac{1}{2}} i-R^{-\frac{1}{2}} A^{\top} E^{*}\right)= \\
& =i^{\top} R i-2 i^{\top} A^{\top} E^{*}+E^{* \top} A R^{-1} A^{\top} E^{*} ;
\end{aligned}
$$

hence, taking again into account the definition of the conductance matrix, $G$, and (21) gives:

$$
i^{\top} A^{\top} E^{*} \leq \frac{1}{2} i^{\top} R i+e^{*}
$$

Therefore,

$$
\dot{V}(e, i) \leq e^{\top} I(e)-\frac{1}{2} i^{\top} R i+e^{*} .
$$

Finally, recalling from the proof of Theorem 1 that $\forall k \in$ $\{1, \ldots, n\}, \phi_{k}^{e} \subset \mathcal{J}_{k}^{e} \Longrightarrow e^{\top} I(e) \leq-e^{\top} D e$, one has that

$$
\dot{V}(e, i) \leq-e^{\top} D e-\frac{1}{2} i^{\top} R i+e^{*} .
$$

The proof then follows similar steps to those of Theorem 1, with

$$
\widehat{\Omega}_{R L C}:=\left\{\left(e^{\top}, i^{\top}\right)^{\top} \in \mathbb{R}^{n+m} ; e^{\top} D e+\frac{1}{2} i^{\top} R i \leq e^{*}\right\}
$$

playing the role of $\widehat{\Omega}$, and $s_{R L C}^{*}$ and $\Omega_{R L C}$ standing, respectively, for $s^{*}$ and $\Omega$.

Corollary 2: Assume that the control current vector $I(e)$ in (16)-(17) is admissible. Then, the network (16)-(17) achieves bounded synchronization with respect to $r(t)=0$. Specifically,

$$
\lim _{t \rightarrow+\infty}\left\|\left(e^{\top}(t), i^{\top}(t)\right)^{\top}\right\| \leq \epsilon_{R L C}=s_{R L C}^{*} \cdot \max \left\{\frac{1}{C_{i}}, \frac{1}{L_{j}}\right\},
$$

with $i=1, \ldots, n, j=1, \ldots, m$.

Remark 2: i) The stability condition for the RLC case is very similar to the one previously obtained for the resistive case. Therefore, the discussion of Remark 1 is also applicable to the present situation if one includes $\frac{L_{k l}}{R_{k l}}$ in the discussion: higher $R_{k l}$ 's and lower $L_{k l}$ 's also reduce the size of $\Omega_{R L C}$. ii) Bounded synchronization is proved both for node voltages, with respect to a reference, and for the transmission line currents, with respect to 0 .

iii) It is worth emphasizing that the results obtained for resistive and RLC systems are applicable to any admissible control law, i.e. according to Definition 2, to any current control vector that places the characteristic curves of every node of the HVDC system within its corresponding $k$-th control admissibility region. It is shown in the next section that this can be fulfilled by means of droop controllers.

\section{APPLICATION}

The numerical simulations presented in this Section consider the RLC case. An example of the resistive case can be found in [34].

An M-VSC HVDC network is considered connecting several wind farms (WF) to grid side (GS) converters located at DC/AC stations in the mainland. The topology is a simplified, 


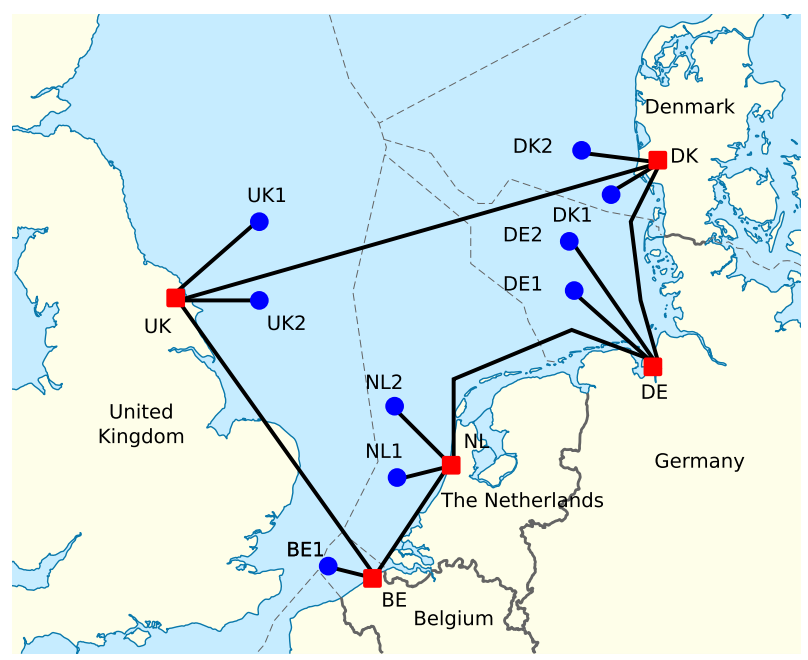

Fig. 8: Scheme of the M-HVDC network representing the North Sea offshore wind integration network used as application example.

meshed version of the offshore wind integration grid in the North Sea [35] with 14 lines and 14 nodes. The network structure is shown in Fig. 8. In each node, a droop control strategy is adopted. As indicated in Subsection III-B, the droop control consists of a nonlinear static relationship between the current provided by the VSC, $I_{k}$, and the voltage across each capacitor, $E_{k}$ (see also the example in [7]).

The droop control in each agent can be generalized as

$$
I_{k}= \begin{cases}I_{k}^{\max } & \text { if } E_{k} \leq P_{k \mathrm{I}} I_{k}^{\max } \\ \frac{E_{k}}{P_{k \mathrm{I}}} & \text { if } P_{k \mathrm{I}} I_{k}^{\max }<E_{k}<E_{k}^{l} \\ -d_{k}^{c}\left(E_{k}-E_{k}^{*}\right) & \text { if } E_{k}^{l}<E_{k}<E_{k}^{h} \\ \frac{E_{k}}{P_{k \mathrm{C}}} & \text { if } E_{k}^{l}<E_{k}\end{cases}
$$

where $P_{k \mathrm{I}}>0$ is the power generated by a WF (or the maximum power that can be delivered by a GS converter in case of supplying energy to the DC network), and $P_{k \mathrm{C}}<0$ is the maximum power that can be consumed by a GS node.
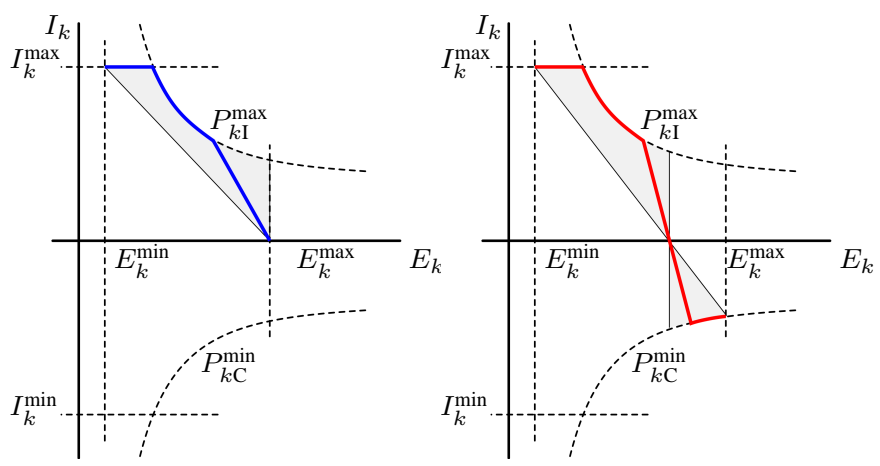

Fig. 9: Droop control curves and control admissibility regions: WF (blue), GS (red).
TABLE I: Simulation node parameters.

\begin{tabular}{|c|c|c|c|c|}
\hline Node & Name & Type & $P_{k}^{\max }$ & $E_{k}^{*}$ \\
\hline N1 & UK1 & WF & $600 \mathrm{MW}$ & $265 \mathrm{kV}$ \\
N2 & UK2 & WF & $400 \mathrm{MW}$ & $265 \mathrm{kV}$ \\
N3 & UK & GS & $850 \mathrm{MW}$ & $245 \mathrm{kV}$ \\
N4 & BE1 & WF & $200 \mathrm{MW}$ & $265 \mathrm{kV}$ \\
N5 & BE & GS & $140 \mathrm{MW}$ & $245 \mathrm{kV}$ \\
N6 & NL1 & WF & $400 \mathrm{MW}$ & $265 \mathrm{kV}$ \\
N7 & NL2 & WF & $200 \mathrm{MW}$ & $265 \mathrm{kV}$ \\
N8 & NL & GS & $540 \mathrm{MW}$ & $245 \mathrm{kV}$ \\
N9 & DE1 & WF & $400 \mathrm{MW}$ & $265 \mathrm{kV}$ \\
N10 & DE2 & WF & $400 \mathrm{MW}$ & $265 \mathrm{kV}$ \\
N11 & DE & GS & $640 \mathrm{MW}$ & $245 \mathrm{kV}$ \\
N12 & DK1 & WF & $200 \mathrm{MW}$ & $265 \mathrm{kV}$ \\
N13 & DK2 & WF & $200 \mathrm{MW}$ & $265 \mathrm{kV}$ \\
N14 & DK & GS & $240 \mathrm{MW}$ & $245 \mathrm{kV}$ \\
\hline
\end{tabular}

TABLE II: Simulation line parameters.

\begin{tabular}{|c|c|c|c|}
\hline Line & Length [km] & Line & Length [km] \\
\hline $\mathrm{L}_{1,3}$ & 100 & $\mathrm{~L}_{12,14}$ & 40 \\
$\mathrm{~L}_{2,3}$ & 120 & $\mathrm{~L}_{13,14}$ & 50 \\
$\mathrm{~L}_{4,5}$ & 100 & $\mathrm{~L}_{3,5}$ & 300 \\
$\mathrm{~L}_{6,8}$ & 100 & $\mathrm{~L}_{5,8}$ & 120 \\
$\mathrm{~L}_{7,8}$ & 40 & $\mathrm{~L}_{12,14}$ & 250 \\
$\mathrm{~L}_{9,11}$ & 40 & $\mathrm{~L}_{12,14}$ & 120 \\
$\mathrm{~L}_{10,11}$ & 70 & $\mathrm{~L}_{12,14}$ & 380 \\
\hline
\end{tabular}

The slope of the droop control is denoted by $d_{k}^{c}$, and $E_{k}^{*}$ is the voltage value for zero injected current. The lower and higher threshold values of the droop region, $E_{k}^{l}$ and $E_{k}^{h}$, can be obtained as

$$
E_{k}^{l, h}=\frac{1}{2}\left(E_{k}^{*} \pm \sqrt{E_{k}^{* 2}-\frac{4 P_{k \mathrm{I}, k \mathrm{C}}}{d_{k}^{c}}}\right)
$$

Notice that for WF power plants the consumed power is zero. Then, for WF nodes, $P_{k \mathrm{C}}=0$ which, taking into account (34), implies $E_{k}^{h}=E_{k}^{*}$.

Notice also that, by construction, droop control laws are admissible in the sense of Definition 2: the curve described by (33) in the $\left(I_{k}, E_{k}\right)$ plane and the corresponding control admissibility region $\mathcal{I}_{k}^{e} \cap \mathcal{J}_{k}^{e}$ are depicted in Fig. 9.

The node parameters are summarized in Table I. In all nodes the capacitances are set to $C_{k}^{v}=75 \mathrm{mF}$, and the droop control slopes have been set to $d_{k}^{c}=2$. The available power in wind farms has been randomly assigned taking a Weibull distribution. For the sake of simplicity, we assume that $P_{k}^{\max }=P_{k \mathrm{C}}=-P_{k \mathrm{I}}$ for GS converters, and $P_{k}^{\max }=P_{k \mathrm{I}}$ for WF nodes. The line lengths are shown in Table II and the resistance and inductance per kilometer are set, from [35], to $R_{u}=0.2 \Omega / \mathrm{km}, L_{u}=19.1 \mathrm{mH} / \mathrm{km}$, and $C_{u}=220 \mathrm{nF} / \mathrm{km}$. The simulation test takes 1000 s and also considers a fault in the GS converter N11 at $t=500 \mathrm{~s}$, when the power is suddenly reduced to $320 \mathrm{MW}$ for $100 \mathrm{~s}$.

In Fig. 10, the available power is displayed at the WF resulting from the random process. It is worth noticing that some nodes (N1, N2, N6 and N10) are underloaded, some are fully loaded (N4 and N7) at all times, and the loads of some other nodes vary with time. The effect of the GS failure is evident in nodes N9 and N10 (the neighbors) and can be also noticed in N12 and N13. 

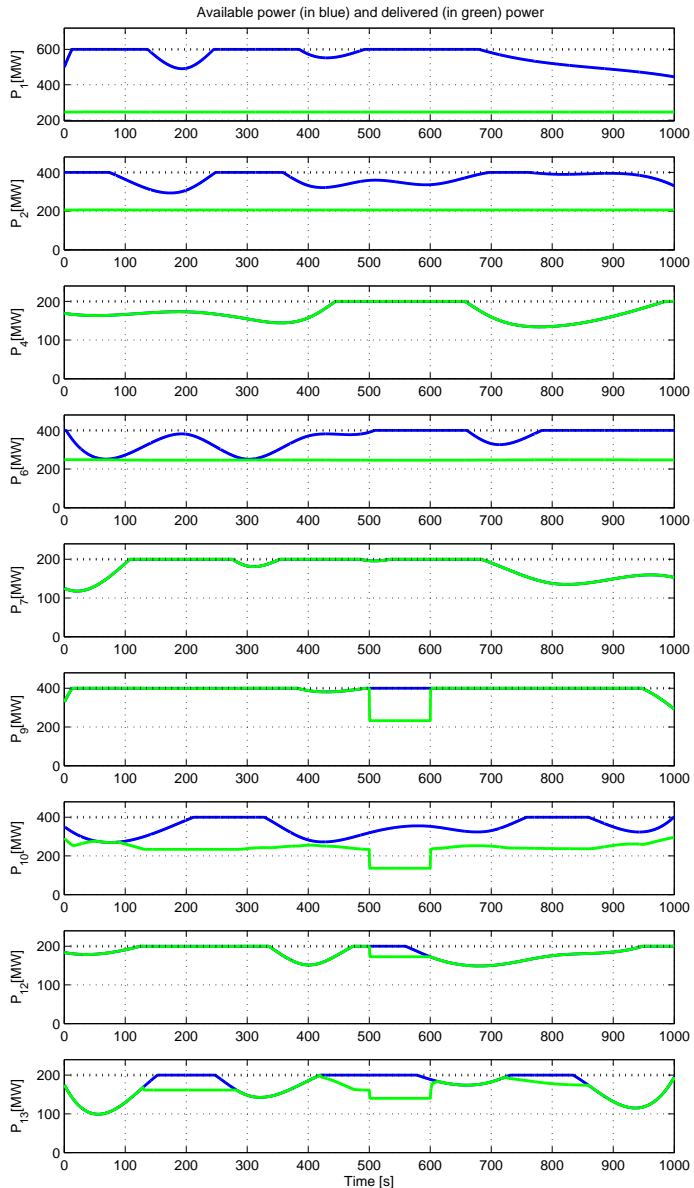

Fig. 10: Instantaneous power at the WF: available (blue) and delivered (green) powers.

The resulting node voltages, $E_{k}$, are displayed in Fig. 11. Notice that, in general, WF nodes (in blue) show higher voltages than GS converters (in red), so that energy flows from generation to consumption/distribution points. It is noticeable that all voltages are bounded by $\max \left(E_{k}\right)$ and $\min \left(E_{k}\right)$. As we will show next, the numerical result is less conservative than the analytical bounds derived in the previous section.

The injected and consumed powers in each WF and GS nodes, respectively, are shown in Fig. 12. Due to wind variability and to the droop control strategy, power in each node is continuously changing to keep the power network within the admissible voltage range.

Fig. 13 shows the droop control strategy for each node. Notice that during the simulations, some nodes are drooping for a certain time while some are kept at the maximum power (N4, N7 and N14)). One can also identify WF and GS nodes because their characteristic curves belong to the

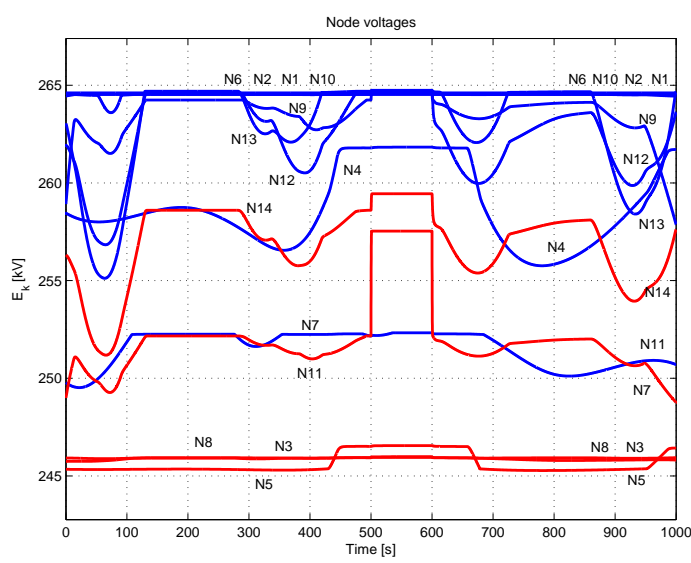

Fig. 11: Node voltages: WF (blue), GS (red).

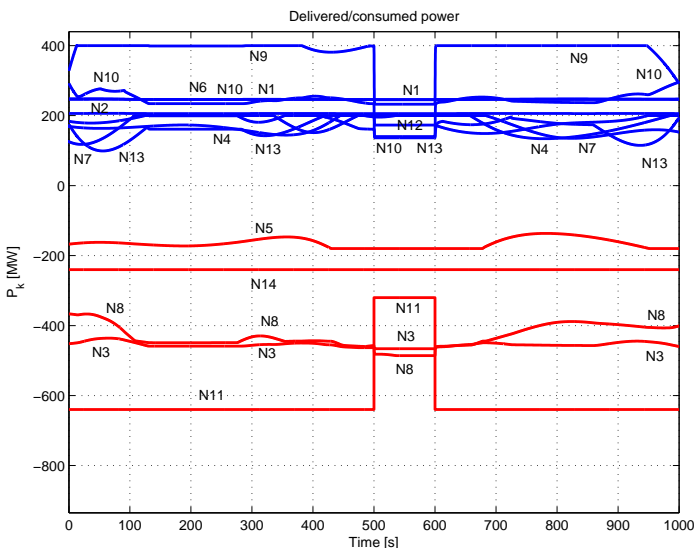

Fig. 12: Instantaneous power: WF (blue), GS (red).

second and fourth quadrant, respectively. The red and black lines correspond to the droop control slope, $d_{k}^{c}$, and to the slopes $d_{k}$ used in the definition of the control admissibility regions $\mathcal{I}_{k}^{e} \cap \mathcal{J}_{k}^{e}$.

Finally, Fig. 14 portrays trajectories projected in some $\left(e_{i}, e_{j}\right)$ planes, together with the projection of the corresponding hyperellipsiod $\Omega_{R L C}$. As the initial conditions are inside $\Omega_{R L C}$ trajectories remain there for all time, as predicted by Theorem 2. Bounded synchronization is therefore achieved, and the bound for $e(t)$ given in equation (32) of Corollary 2 amounts to $\epsilon=73.0 \mathrm{kV}$; this corresponds, approximately, to $25 \%$ of the nominal value.

Summarizing, this numerical test not only validates the theoretical results derived in Section $\mathrm{V}$, but also shows that, in accordance with them, the droop control strategy bounds the DC voltages in VSC-HVDC networks.

\section{CONCLUSIONS}

In this paper we presented a complex networks-based approach to study multi-terminal VSC-HVDC resistive and RLC 

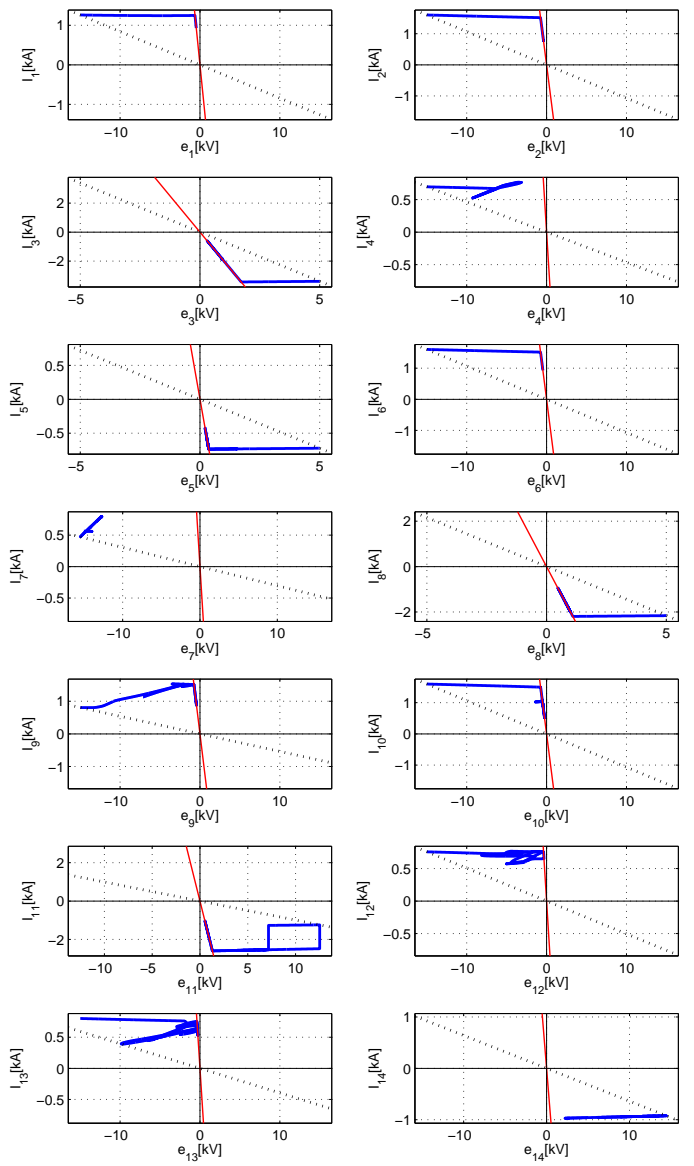

Fig. 13: Droop control action in the $\left(e_{k}, I_{k}\right)$ plane for each node. Red straight line: droop control slope, $d_{k}^{c}$; black dotted line: minimum value for $d_{k}$ that defines the $k$-th control admissibility region.

transmission lines using a complex network approach. Firstly, the system was modelled as a complex dynamical network, the currents of the VSC converters of each terminal being control variables. Then, bounded synchronization of the node voltages was proved to occur for a family of admissible controllers, i.e. control laws able to place the characteristic curve of each node in a certain region, and explicit synchronization bounds were computed as well. The commonly used droop control strategy was also shown to be an admissible control. Finally, the analytical results were successfully validated via numerical simulations.

The fact that the derivations were made for a generic family of controllers yielded conservative synchronization bounds. Hence, further research should address design issues, namely, the obtaining of control laws that place the system in a pre-assigned, thus more convenient from the synchronization
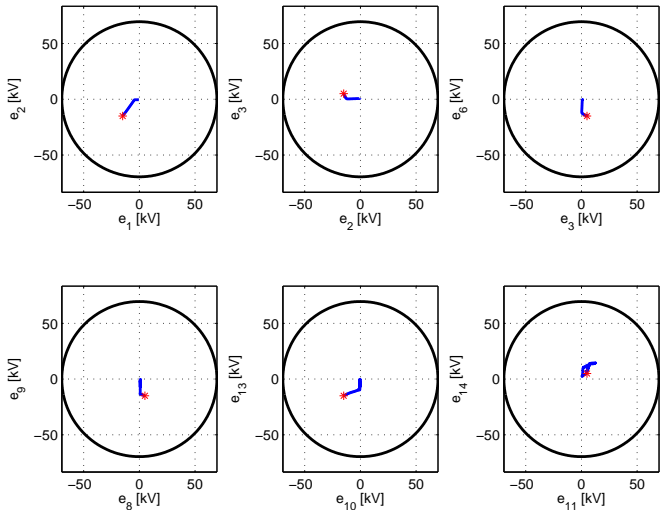

Fig. 14: Projected trajectories and $\Omega_{R L C}$ in some $\left(e_{i}, e_{j}\right)$ planes.

bounds side, control admissibility region. Also of interest is to take into account the current controller dynamics (see Fig. 2): a first order model of it [7] would result in a complex network with two-dimensional nodes and state variables $\left(E_{k}, I_{k}\right)$.

\section{REFERENCES}

[1] N. Flourentzou, V. Agelidis, and G. Demetriades, "VSC-based HVDC power transmission systems: An overview," IEEE Trans. on Power Electronics, vol. 24, no. 3, pp. 592-602, 2006.

[2] D. van Hertem and M. Ghandhari, "Multi-terminal VSC HVDC for the European supergrid: Obstacles," Renewable \& Sustainable Energy Reviews, vol. 14, no. 9, pp. 3156-3163, 2010.

[3] M. Bahrman, "HVDC transmission overview," in Proc. Transmission and Distribution Conference and Exposition, 2008, pp. 1-7.

[4] C. Chou, Y. Wu, G. Han, and C. Lee, "Comparative evaluation of the HVDC and HVAC links integrated in a large offshore wind farm an actual case study in Taiwan," IEEE Trans. Industry Applications, vol. 48, no. 5, pp. 1639-1648, 2012.

[5] E. Prieto-Araujo, F. Bianchi, A. Junyent-Ferré, and O. Gomis-Bellmunt, "Methodology for droop control dynamic analysis of multiterminal VSC-HVDC grids for offshore wind farms," IEEE Trans. on Power Delivery, vol. 26, no. 4, pp. 2476-2485, 2011.

[6] O. Gomis-Bellmunt, J. Liang, J. Ekanayake, and N. Jenkins, "Voltagecurrent characteristics of multiterminal HVDC-VSC for offshore wind farms," Electric Power Systems Research, vol. 81, no. 2, pp. 440-450, 2011.

[7] A. Egea-Alvarez, F. Bianchi, A. Junyent-Ferré, G. Gross, and O. GomisBellmunt, "Voltage control of multiterminal VSC-HVDC transmission systems for offshore wind power plants: design and implementation in a scaled platform," IEEE Trans. on Industrial Electronics, vol. 60, no. 6, pp. 2381-2391, 2013.

[8] F. Bianchi and O. Gomis-Bellmunt, "Droop control design for multiterminal VSC-HVDC grids based on LMI optimization," in Proc. 50th IEEE Conf. on Decision and Control, 2011, pp. 4823-4828.

[9] S. Boccaletti, V. Latora, Y. Moreno, M. Chavez, and D. Hwang, "Complex networks: Structure and dynamics," Physics Reports, vol. 424, no. 4-5, pp. 175-308, 2006.

[10] M. Newman, "The structure and function of complex networks," SIAM Review, vol. 45, pp. 167-256, 2003. 
[11] R. Olfati-Saber, J. Fax, and R. Murray, "Consensus and cooperation in networked multi-agent systems," Proc. of the IEEE, vol. 95, no. 1, pp. 215-233, 2007

[12] E. Nuño, R. Ortega, L. Basañez, and D. Hill, "Synchronization of networks of nonidentical Euler-Lagrange systems with uncertain parameters and communication delays," IEEE Trans. on Automatic Control, vol. 56, no. 4, pp. 935-941, 2011.

[13] J. Zhao, D. Hill, and T. Liu, "Global bounded synchronization of general dynamical networks with nonidentical nodes," IEEE Trans. Automatic Control, vol. 57, no. 10, pp. 2656-2662, 2012.

[14] C. Murguia, R. Fey, and H. Nijmeijer, "Synchronization of identical linear systems and diffusive time-delayed couplings," IEEE Trans. Circuits and Systems I: Regular Papers, vol. 61, no. 6, pp. 1801-1814, 2014.

[15] T. Liu, D. Hill, and J. Zhao, "Output synchronization of dynamical networks with incrementally-dissipative nodes and switching topology," IEEE Trans. Circuits and Systems I: Regular Papers, vol. 62, no. 9, pp. 2312-2323, 2015.

[16] Z. Li, Z. Duan, G. Chen, and L. Huang, "Consensus of multiagent systems and synchronization of complex networks: A unified viewpoint," IEEE Trans. Circuits and Systems I: Regular Papers, vol. 57, no. 1, pp. 213-224, 2010.

[17] W. Yu, G. Chen, W. Ren, J. Kurths, and W. Zheng, "Distributed higher order consensus protocols in multiagent dynamical systems," IEEE Trans. Circuits and Systems I: Regular Papers, vol. 58, no. 8, pp. 19241932, 2011.

[18] P. DeLellis, M. di Bernardo, and D. Liuzza, "Convergence and synchronization in heterogeneous networks of smooth and piecewise smooth systems," Automatica, vol. 56, pp. 1-11, 2015.

[19] G. Pagani and M. Aiello, "The power grid as a complex network: A survey," Physica A, vol. 392, pp. 2688-2700, 2013.

[20] Y. Uwate and Y. Nishio, "Synchronization in several types of coupled polygonal oscillatory networks," IEEE Trans. Circuits and Systems I: Regular Papers, vol. 59, no. 5, pp. 1042-1050, 2012.

[21] F. Dörfler, M. Chertkov, and F. Bullo, "Synchronization in complex oscillator networks and smart grids," Proc. National Academy of Sciences, vol. 110, no. 6, pp. 2005-2010, 2013.

[22] A. Motter, M. A. S.A. Myers, and T. Nishikawa, "Spontaneous synchrony in power-grid networks," Nature Physics, vol. 9, pp. 191-197, 2013.

[23] J. Schiffer, D. Goldin, J. Raisch, and T. Sezi, "Synchronization of droop-controlled microgrids with distributed rotational and electronic generation," in Proc. 52nd IEEE Conf. on Decision and Control, 2013.

[24] J. Simpson-Porco, F. Dörfler, and F. Bullo, "Synchronization and power sharing for droop-controlled inverters in islanded microgrids," Automatica, vol. 49, no. 9, pp. 2603-2611, 2013.

[25] E. Hancock and D. Hill, "Restricted partial stability and synchronization," IEEE Trans. Circuits and Systems I: Regular Papers, vol. 61, no. 11, pp. 3235-3244, 2014.

[26] B. Johnson, S. Dhople, A. Hamadeh, and P. Krein, "Synchronization of nonlinear oscillators in an LTI power network," IEEE Trans. Circuits and Systems I: Regular Papers, vol. 61, no. 3, pp. 834-844, 2014.

[27] S. Dhople, B. Johnson, F. Dörfler, and A. Hamadeh, "Synchronization of nonlinear circuits in dynamic electrical networks with general topologies," IEEE Trans. Circuits and Systems I: Regular Papers, vol. 61, no. 9, pp. 2677-2690, 2014.

[28] A. Maknouninejad, Z. Qu, F. Lewis, and A. Davoudi, "Optimal, nonlinear, and distributed designs of droop controls for DC microgrids," IEEE Trans. Smart Grid, vol. 5, no. 5, pp. 2508-2516, 2014.

[29] D. Zonetti, R. Ortega, and A. Benchaib, "Modeling and control of HVDC transmission systems from theory to practice and back," Control Engineering Practice, vol. 45, pp. 133-146, 2015.

[30] F. Dörfler and F. Bullo, "Kron reduction of graphs with applications to electrical networks," IEEE Trans. Circuits and Systems I: Regular Papers, vol. 60, no. 1, p. 150163, 2013.
[31] S. Caliskan and P. Tabuada, "Towards Kron reduction of generalized electrical networks," Automatica, vol. 50, no. 10, pp. 2586-2590, 2014.

[32] P. DeLellis, M. diBernardo, F. Garofalo, and M. Porfiri, "Evolution of complex networks via edge snapping," IEEE Trans. Circuits and Systems I: Regular Papers, vol. 57, no. 8, pp. 2132-2143, 2010.

[33] H. Sayama, I. Pestov, J. Schmidt, B. Bush, C. Wong, J. Yamanoi, and T. Gross, "Modeling complex systems with adaptive networks," Computers \& Mathematics with Applications, vol. 65, no. 10, p. $16451664,2013$.

[34] A. Dòria-Cerezo, J. Olm, M. di Bernardo, M. Quaglia, and E. Nuño, "Bounded synchronization in resistive multiterminal VSC-HVDC transmission systems," in Proc. 53th IEEE Conf. on Decision and Control, 2014, pp. 3677-3682.

[35] S. Rodrigues, R. Teixeira-Pinto, P. Bauer, and J. Pierik, "Optimal power flow control of VSC-based multiterminal DC networks offshore wind integration in the North Sea," IEEE Journal of Emerging and Selected Topics in Power Electronics, vol. 1, no. 4, pp. 260-268, 2013.

\section{ACKNOWLEDGEMENTS}

A. Dòria-Cerezo and J.M. Olm are partially supported by the spanish Ministerio de Educación project DPI2013-41224-P and the catalan AGAUR project 2014 SGR 267. 